\title{
Ética de la asistencia primaria a los niños de procedencia internacional ${ }^{1}$
}

Vicente Bellver Capella ${ }^{2}$

Resumen: La inmigración se ha convertido en un fenómeno universal. Uno de sus efectos es el incremento de los niños de procedencia internacional en las consultas de pediatría. Se trata de una población doblemente vulnerable, por tratarse de niños y por proceder de contextos culturales extraños a los del país de acogida. Ante esta realidad emergente es imprescindible preguntarse por el tipo de asistencia sanitaria que deben recibir. En el presente artículo se proponen principios éticos de actuación inspirados en las principales normas internacionales sobre bioética y derechos de los niños: principio de vulnerabilidad; principio de respeto y amor, y principio de acceso y atención sanitaria. Finalmente, se proponen algunas políticas que las administraciones podrían (deberían) implementar para garantizar una asistencia pediátrica correcta a los niños inmigrantes.

Palabras clave: asistencia pediátrica, bioética, inmigración

\section{Ethics of primary health care for children of international origin}

Abstract: Immigration has become an universal phenomenon. One of its effects is the increase in children of international origin in pediatric consultation. This is a doubly vulnerable population, because of being children and because of belonging to cultural contexts foreign to host country. Considering this emergent reality it is essential to question the type of health care they must receive. In the present study, ethical principles for acting are proposed inspired in the main international guidelines about bioethics and children rights: vulnerability principle; respect and love principle; and the principle of access to health care. Finally, some policies that health care managements could implement are proposed to guaranty fair pediatric health care for immigrant children.

Key Words: pediatric health care, bioethics, immigration

\section{Ética da assistência primária às crianças de procedência internacional}

Resumo: A imigração se converteu num fenômeno universal. Um de seus efeitos é o incremento das crianças de procedência internacional nas consultas de pediatria. Trata-se de uma população duplamente vulnerável por se tratar de crianças e por proceder de contextos culturais estranhos aos do país de acolhida. Ante esta realidade emergente é imprescindível perguntarse pelo tipo de assistência sanitária que devem receber. No presente artigo se propóem princípios éticos de atuação inspirados nas principais normas internacionais sobre bioética e direitos das crianças: princípio de vulnerabilidade; princípio de respeito e amor, e princípio de acesso e atenção sanitária. Finalmente, se propóem algumas políticas que as administrações poderiam (deveriam) implementar para garantir uma assistência pediátrica correta às crianças imigrantes.

Palavras-chave: assistência pediátrica, bioética, imigração

\footnotetext{
${ }^{1}$ Este trabajo se ha realizado dentro del Proyecto DER2010-17357/J URI, financiado por el Ministerio de Educación de Espańa ${ }^{2}$ Profesor titular de Filosofía del Derecho y Filosofía Política, Universitat de València, España

Correspondencia: vicente.bellver@uv.es
} 


\section{Introducción}

Cada vez es más frecuente que acudan niños de procedencia internacional a las consultas pediátricas de atención primaria. La mayoría provienen de la inmigración, pero también llegan niños adoptados de otros países. Aunque este fenómeno ha irrumpido con fuerza en la última década en los llamados países desarrollados, no es exclusivo de esas regiones. Cualquier Estado que no blinde sus fronteras y que ofrezca estabilidad política y posibilidades de empleo, se convierte automáticamente en destinatario de la inmigración. La globalización no solo ha incrementado los flujos migratorios sino también su variabilidad: en periodos de tiempo relativamente cortos, regiones receptoras pueden pasar a emisoras y viceversa.

Se podría pensar que, para la adecuada asistencia sanitaria de los niños de procedencia internacional, basta con conocer las patologías específicas que puedan desarrollar o traer consigo $(1,2)$. Siendo imprescindible ese conocimiento, será sin duda insuficiente si no se desenvuelve dentro de unas bases éticas que garanticen la corrección de la asistencia pediátrica a estos niños $(3,4)$. Lo mismo sucede con los niños nacionales: si la asistencia sanitaria no está informada por unas bases éticas razonables, los resultados que se obtendrán serán menos eficaces o incluso contraproducentes. Ahora bien, las circunstancias que rodean al niño inmigrante exigen un abordaje ético específico.

En estas páginas me ocuparé de los aspectos éticos de la asistencia primaria a los menores de procedencia internacional. No trataré de las cuestiones que suscita la asistencia hospitalaria. La asistencia primaria suele ser, para la mayoría de los niños, la puerta de acceso y el contacto más continuo con el sistema sanitario. Tiene un influjo decisivo en su desarrollo y en la visión que tendrán de su salud y de la asistencia sanitaria, y por ello, merece una atención particular $(5,6)$. Entre los niños de procedencia internacional centraré la atención en los inmigrantes por razones económicas o humanitarias, que son los más frecuentes, y los que se encuentran en una situación de mayor vulnerabilidad.

Cuando se trata de los aspectos éticos de la asistencia pediátrica primaria se suele pensar en cues- tiones relacionadas con el menor maduro y sus derechos a la confidencialidad, la intimidad, la información y el consentimiento informado. Estas cuestiones merecen, sin duda, una atención minuciosa, para ofrecer respuestas correctas que se aparten tanto del paternalismo/proteccionismo como del primado absoluto de la voluntad del menor. Pero la asistencia pediátrica primaria tiene un campo mucho más amplio, que más que a discutir sobre casos difíciles invita a descubrir caminos de excelencia(7).

Es frecuente que la bioética pediátrica se centre en la figura del pediatra y apenas tenga en cuenta a las enfermeras $(8,9)$. Nos parece un error grave. Por más que las inercias y los intereses se empeñen en mantener una jerarquía en la asistencia, en cuya cúspide se encontraría el pediatra, lo cierto es que la asistencia cotidiana alcanza su mejor expresión cuando existe una estrecha colaboración entre el pediatra y la enfermera, sin que esta última se limite a ejecutar funciones subalternas o a proporcionar información. Por las consultas pediátricas de atención primaria circula una importante proporción de niños sanos, y la enfermera tiene un papel decisivo en las visitas periódicas que hacen estos niños durante sus primeros años de vida, para seguir la evolución de su desarrollo, cambiar la dieta, aplicar el programa de vacunación, etc.

Pero antes de proponer los principios éticos que deberían informar la asistencia primaria a los nińos de la inmigración parece oportuno referirse a los dos aspectos que determinan la especificidad de la relación entre los niños de procedencia internacional y el personal sanitario: primero, que esas personas son niños, no mayores de edad; segundo, que el niño trae consigo una cultura que no es la del país en el que ahora vive.

\section{Ética, infancia y diversidad cultural}

Que la asistencia pediátrica maneje correctamente cada uno de los dos elementos mencionados no es sencillo. Por el contrario, se suele incurrir en posiciones extremas, igualmente injustas. Veamos los errores que se derivan de no dar a la niñez ni a la diversidad cultural el peso adecuado en la relación pediátrica. 
La atención a menores: entre el paternalismo y el autonomismo

El modelo de relación entre profesionales y usuarios del sistema de salud ha sufrido grandes cambios en las últimas décadas y, si bien se han producido importantes progresos, tampoco se puede desconocer la perplejidad en la que se encuentran tanto unos como otros(10). Porque en este ámbito se han adoptado posiciones extremas que atentan no solo contra los pacientes sino contra los mismos profesionales. Esta situación se agrava cuando nos centramos en la atención sanitaria a los menores.

Resulta difícil compartir la visión simplista que, sin embargo, se suele aceptar como verdad oficial, según la cual el modelo de relación entre los profesionales sanitarios y los pacientes ha pasado del paternalismo al autonomismo. De una relación de dominación se habría pasado a otra de igualdad. Si esta relación tenía aspectos negativos en el pasado, también los tiene en la actualidad. En lugar de insistir en lo mal que se hacían las cosas en el pasado y en lo bien que se hacen ahora, sería más conveniente denunciar los problemas que presenta la relación sanitaria en la actualidad y ofrecer vías de solución. Es indudable que se han hecho progresos en ciertos aspectos, pero aún falta mucho por hacer y, lo que es más grave, en otros aspectos incluso se ha retrocedido.

Si la cuestión es compleja cuando el paciente es un adulto, más aún lo es cuando se trata de un menor. Los principales errores en este campo proceden de dar el protagonismo exclusivo solo a uno de los integrantes de la relación: bien a los padres, al niño o al profesional sanitario. Lo lógico es que los tres participen en la toma de decisiones, modulando el peso de su participación en función de la capacidad del menor para asumir sus propias decisiones. Así se sorteará tanto el riesgo del paternalismo de los profesionales o de los padres como el autonomismo del menor(11). No es fácil dar con la respuesta acertada en cada caso, y menos cuando los menores que reciben asistencia sanitaria son de origen internacional y pueden proceder, en consecuencia, de contextos culturales completamente ajenos al nuestro. En esos casos habrá que estar igualmente dispuestos a respetar posiciones que no nos parezcan acertadas, siem- pre que no atenten contra los derechos del menor, y a defender los derechos de éste frente a entornos familiares y culturales que traten de sofocarlos.

\section{La atención a la diversidad cultural: entre el etno- centrismo y el multiculturalismo}

Un profesional de la sanidad percibe la importancia del pluralismo cultural en el mundo actual a través de los medios de comunicación, de su experiencia como ciudadano y, sobre todo, de su trabajo en la consulta. Allí se encuentra con gentes de las más diversas procedencias, etnias, culturas y religiones. Y es allí donde se le plantean, a través de casos concretos, los problemas de fondo generados por la globalización y el pluralismo cultural(12). A la hora de tratarlos, comparecen con frecuencia dos posiciones extremas, que tienen algo de dogmáticas y que en absoluto resultan adecuadas para dar con la respuesta correcta.

El etnocentrismo. Es la posición de las sociedades que niegan cualquier reconocimiento a los inmigrantes que deseen mantener formas de vida que no se compadezcan enteramente con las de la cultura dominante en esa sociedad. Al impedir a los inmigrantes la posibilidad de vivir de acuerdo con aspectos fundamentales de su cultura, se les niega la posibilidad de su desarrollo personal. Podríamos decir que se pretende que los niños desarrollen su personalidad pero a nuestra manera. Lógicamente, la arbitrariedad se trata de camuflar tras razones de salud: "debe hacer esto y no deber hacer lo otro porque es lo que conviene a su salud". Este modo de actuar desconoce que la salud, si bien tiene un valor universal, es al mismo tiempo un concepto histórico y cultural, que varía en función de los territorios y los tiempos. Pretender que el concepto de salud que manejamos en nuestra sociedad es el único válido, con carácter universal, es demasiado pretencioso, y tratar de imponerlo a los niños internacionales y a sus padres es una injerencia en la libertad de unos y otros.

El multiculturalismo. Consiste en dar por buena la diversidad cultural en sí misma. No se entra en valoraciones sobre el contenido de cada cultura y se da por supuesto que la convivencia en paz queda asegurada tolerando el florecimiento de todas ellas, siempre que cada una respete a las demás. 
Esta posición, como la anterior, irrespeta a la persona al dar preferencia a la ordenación impuesta por cada cultura sobre los derechos humanos universales. Además, incurre en la ingenuidad de pensar que la simple agregación de grupos culturales diversos da lugar a un orden social justo y pacífico. La salud y la enfermedad tienen mucho de constructo cultural, pero no se disuelven enteramente en la cultura, de modo que se pueden reconocer elementos de alcance universal que deben ser tenidos siempre en cuenta.

\section{Principios éticos de la atención pediátrica en niños internacionales}

Establecido el marco en el que se debería desenvolver la ética de la asistencia pediátrica a los niños de procedencia internacional, propondré los principios de actuación para los profesionales que se dedican a esta área sanitaria tan sensible y fundamental.

Estos principios están inspirados en los textos normativos más relevantes aprobados internacionalmente sobre los derechos de los niños y sobre bioética. Respecto de los primeros, tenemos la Declaración de Derechos del Niño (1959), que dio lugar treinta años más tarde a la Convención sobre Derechos del Niño (1989), aprobadas ambas por la Asamblea General de Naciones Unidas. En relación con la segunda destacan la mencionada Declaración Universal sobre Derechos Humanos y Bioética (2005) y la Convención Europea sobre Derechos Humanos y Biomedicina (1996).

Estos documentos sintetizan el acuerdo universal acerca del trato que debemos a los niños hasta los 18 años en el ámbito sanitario. Reflejan, por tanto, los valores y principios compartidos por las grandes culturas vigentes en el mundo.

Son tres los principios a tener en cuenta:

\section{El principio de vulnerabilidad}

"Los seres humanos somos vulnerables a una gran cantidad de aflicciones diversas, entre estas el padecer alguna enfermedad grave en uno u otro momentos de la vida"(13:15). Estas palabras de MacIntyre son particularmente ciertas en el caso de los niños. Y no digamos si se trata de niños de procedencia extranjera que deben recibir asistencia sanitaria. En ellos pueden llegar a concurrir hasta seis condiciones que incrementan su vulnerabilidad(14).

- Infancia. Si todo ser humano es vulnerable, existen franjas de edad en las que esa vulnerabilidad es mayor, particularmente la infancia y la ancianidad. Los niños no solo son vulnerables porque las capacidades propias del ser adulto todavía no se han desarrollado de forma suficiente - la racionalidad y la libertad-, sino sobre todo porque los cuidados que reciban de los demás van a determinar el grado de desarrollo que alcanzarán, tanto físico, como psíquico, cognitivo, afectivo, etc.

- Enfermedad. En el caso de los niños, la enfermedad evidencia su debilidad con mayor claridad aún que en los adultos, porque, si bien tienen una extraordinaria capacidad de recuperación, también las secuelas de la enfermedad pueden tener un alcance mucho mayor; porque el niño no tiene capacidad, en muchas ocasiones, de tomar por sí mismo las decisiones sobre su atención sanitaria, y porque parece un sinsentido que la enfermedad, sobre todo cuando es grave y prolongada, se cebe en personas a las que les correspondería crecer felices.

- Extranjería. Es cierto que los niños inmigrantes y adoptados vienen a los países de acogida con el fin de disfrutar de unas condiciones de vida mejores que las de sus países de origen. Pero también lo es que el cambio produce un profundo desarraigo, al variar el entorno cultural en el que se habían criado hasta entonces: la lengua, el territorio y el paisaje, las costumbres, la alimentación, la religión, las relaciones sociales, entre otros.

- Pobreza. Normalmente de esta carga suelen librarse tanto los nińos adoptados como los procedentes de la inmigración y que ya han arraigado en el país o vinieron por razones distintas a la necesidad. Pero son muchos los niños inmigrantes que traen consigo la pobreza, y la mantienen o incluso profundizan en ella en el país de acogida. Esta circunstancia es compartida por los niños pobres nacio- 
nales, solo que en los de procedencia internacional concurren los otros elementos que incrementan su vulnerabilidad.

- Desestructuración familiar. Aunque la inmigración puede ofrecer oportunidades de desarrollo personal y familiar, de entrada y en casi todos los casos trae consigo una situación de fuerte estrés en toda la familia, cuyas principales manifestaciones son: la ruptura de la unidad familiar, en los casos en los que parte de la familia se queda en el país de origen; la inestabilidad familiar producida por el cambio del contexto, y la reducción del tiempo dedicado a la atención de los hijos, porque frecuentemente tienen que trabajar durante largas jornadas los dos miembros de la pareja o el único que ha emigrado con los niños. Todo ello tiene una enorme repercusión sobre éstos, que pueden perder las referencias, la dedicación y el afecto que necesitan para avanzar en su desarrollo personal.

- Discapacidad. En las últimas décadas, sobre todo en los países más desarrollados, se ha avanzado mucho en conseguir la igualdad efectiva de oportunidades para las personas con discapacidad. Se han potenciado especialmente las políticas públicas para las personas con discapacidad física o sensorial. La discapacidad psíquica está, en cambio, más lejos de obtener la atención necesaria para conseguir que todas las personas que la padecen tengan un horizonte de oportunidades. En cualquier caso, cuando son niños los que la padecen se disparan los riesgos de que su desarrollo personal se vea truncado.

- La infancia femenina. Todas las anteriores circunstancias se suelen agravar, más si cabe, cuando quienes las sufren son niñas. Están expuestas a un mayor riesgo de explotación en todos los ámbitos en los que se desenvuelven: desde el familiar hasta el laboral. A partir de la edad en la que ya pueden ser madres aparece el riesgo de agresión sexual y embarazo no deseado que las convierten probablemente en el prototipo de niña vulnerable.

Decía MacIntyre que el modo en que el ser humano sobrevive y aun florece en medio de la vul- nerabilidad depende solo en una pequeña medida de sí mismo(13). En el caso de los niños podríamos decir que esa dependencia es máxima. De ahí que el principio consagrado universalmente sobre el trato debido a los niños sea el del interés superior del menor.

La Declaración Universal sobre Bioética y Derechos Humanos, aprobada por la UNESCO en 2005, hace referencia expresa a la vulnerabilidad. En su artículo 8, dedicado al respeto de la vulnerabilidad humana y la integridad personal, dice: "Al aplicar y fomentar el conocimiento científico, la práctica médica y las tecnologías conexas, se debería tener en cuenta la vulnerabilidad humana. Los individuos y grupos especialmente vulnerables deberían ser protegidos y se debería respetar la integridad personal de dichos individuos". Este artículo de la Declaración deberá ser tenido especialmente en cuenta por los profesionales sanitarios en su asistencia a los niños de procedencia internacional.

\section{El principio de respeto y amor por los niños}

El primer texto normativo de alcance internacional sobre los derechos de los niños estableció un mandato sumamente exigente: "la humanidad debe dar al niño lo mejor de sí misma" (Declaración de Ginebra de 1924 sobre los derechos del niño). En esa línea de exigencia, la Declaración de 1959, tan breve como contundente en la proclamación de cada uno de sus principios, afirmó: "El niño, para el pleno desarrollo de su personalidad, necesita amor y comprensión. Siempre que sea posible, deberá crecer al amparo y bajo la responsabilidad de sus padres y, en todo caso, en un ambiente de afecto y de seguridad moral y material..." (Principio 6). Desde una perspectiva estrictamente legal, la propuesta podría parecer deficiente, porque no se puede exigir bajo coacción que los niños reciban amor y comprensión. Y sin embargo tendemos a darla por buena porque si existe una circunstancia que justifique llevar el Derecho hasta el límite de sus posibilidades, exigiendo lo que es coactivamente inexigible, es el cuidado que los niños requieren para su desarrollo. La Convención sobre los Derechos del Niño, en lugar de incluir esta exigencia en su texto articulado, lo hizo en el Preámbulo — que no tiene valor normativo pero sí declarativo-, en los 
siguientes términos: "Reconociendo que el niño, para el pleno y armonioso desarrollo de su personalidad, debe crecer en el seno de la familia, en un ambiente de felicidad, amor y comprensión”.

¿Qué consecuencias para la atención sanitaria de los niños internacionales se derivan de este doble principio? Destacan dos:

- Si cualquier profesional sanitario tiene el deber de ganarse la confianza de las personas a las que trata, ese deber se agrava y adquiere un perfil específico cuando esas personas son niños. El profesional debe adoptar una actitud abiertamente afectiva, que puede incluir el juego en función de la edad de los niños a los que trate. Parte fundamental de la atención consistirá en familiarizarle con un entorno que, de entrada, se le aparece como extraño y amenazante. Tanto el niño como la familia tienen que percibir que son parte activa del equipo de salud y no puros sujetos pasivos. Los encuentros del médico y la enfermera con el niño y la familia deben ser aprovechados para avanzar en la educación para la salud, en el amplio marco de respeto a la diversidad cultural, del que se hablará más adelante.

- Los profesionales sanitarios deben prestar una asistencia que contribuya a la armonía del grupo familiar, en la medida en que esta sea garantía del buen desarrollo del niño. Pueden darse casos de enfrentamientos entre niños y padres sobre la asistencia sanitaria a los primeros. En esos casos, por lo general infrecuentes y siempre complejos, el profesional priorizará el interés del menor, lo que implicará actuaciones que no profundicen en el conflicto sino que tiendan a la conciliación. En la generalidad de los casos los profesionales pueden adoptar una actitud en la que simultáneamente se ayude al niño a que vaya madurando y formando su propio juicio, y se refuerce el papel de la familia en la consecución de ese objetivo.

La infancia es el tiempo en el que, desde la completa dependencia inicial respecto de los adultos, se va desarrollando paulatinamente la capacidad moral del individuo. Esta situación obliga a favo- recer el desarrollo progresivo de la autonomía del menor. Ahora bien, para tener una visión completa del cuadro es necesario tomar en consideración otros dos elementos. Primero, que las distintas etapas de la infancia tienen valor por sí mismas y no solo por ser pasos necesarios hacia la vida autónoma. De ahí que sea tan importante pensar en el futuro como en el presente del niño. Y, segundo, que la autonomía solo crece cuando existen unas raíces identitarias profundas. Por ello, es fundamental para el desarrollo infantil el respeto a sus señas de identidad, que principalmente proceden del ámbito familiar. En consecuencia, la asistencia sanitaria tiene que ser respetuosa con la familia y la cultura en y de la que vive el menor, siempre que no atente contra su dignidad y derechos.

\section{El principio de acceso y asistencia sanitaria compe- tente y respetuosa con la diversidad cultural}

El niño de procedencia internacional presenta notables diferencias en cuanto a su estado de salud y desarrollo con relación al de procedencia nacional. Las más destacables son las siguientes:

- La etnia, e incluso el ambiente en que se ha criado el niño hasta entonces, traen consigo unos patrones de desarrollo distintos de los del país de acogida.

- El niño puede haber importado enfermedades con poca o nula incidencia en el país de acogida, para las cuales los profesionales no han recibido un entrenamiento específico.

- El entorno cultural del niño puede fomentar hábitos claramente insalubres o al menos tenidos por tales si se siguen los parámetros de salud vigentes en el país de acogida.

- Es frecuente que los niños de origen internacional procedan de ambientes de pobreza y marginalidad, y que no lleguen a salir de ellos una vez en el país de acogida. En este caso, no suelen existir diferencias relevantes entre los problemas de salud de los niños nacionales e internacionales.

- El desarraigo que sufren los inmigrantes tiene sus indudables manifestaciones en problemas de salud y, en particular, de salud mental. 
Particular atención requerirá el impacto de la disgregación familiar en el niño, en los casos en los que se produce.

Todas estas diferencias exigen una respuesta sanitaria también diferenciada. No se trata de dar a los niños de procedencia internacional una asistencia privilegiada, sino ajustada a sus necesidades de salud. Para ello los profesionales deberán adquirir las competencias precisas en dos ámbitos distintos pero estrechamente relacionados: los cuidados sanitarios, tanto médicos como de enfermería, y el adecuado manejo de los hábitos y actitudes culturalmente extraños que traen consigo los jóvenes pacientes y sus familias.

Pero así como la información estrictamente sanitaria se puede recabar con facilidad e incorporar a la asistencia sanitaria con prontitud, la generación de hábitos adecuados para tratar personas con culturas distintas, y en ocasiones notablemente extrañas para nosotros, exige más deliberación, formación y, en definitiva, tiempo. En función de la teoría de la justicia con la que el profesional se sienta más familiarizado, así será la atención sanitaria que prestará a los niños internacionales. Ahora bien, no todas son igualmente correctas. Por ejemplo, un médico puede tender a ofrecer una asistencia diferenciada en función de la identidad cultural del niño, aunque ello suponga un incremento de tiempo o recursos, mientras que otro puede tender a considerar irrelevante la diferencia cultural y proporcionar, en consecuencia, el mismo tiempo y recursos al niño inmigrante que a cualquier otro. ¿Qué actitud es la correcta? En principio, parece claro que la primera. Pero no hay que perder de vista que los recursos son limitados y que la adecuada atención al niño inmigrante no puede ir en perjuicio de la del nacional. Este tipo de controversias no tienen por qué suscitarse, pero ponen de manifiesto que los problemas de justicia distributiva (¿qué atención sanitaria es debida a cada uno?) son mucho más complejos que los relativos a la asistencia sanitaria, que se resuelven con más de estudio y entrenamiento.

Para colmo, la reflexión teórica no es suficiente para garantizar una asistencia sanitaria adecuada a la diversidad cultural, porque lo que resulte de ella debe ser llevado a la práctica y, para lograrlo, es imprescindible que los profesionales tengan los hábitos morales idóneos para hacerlo. Por ejemplo, si uno siente un rechazo espontáneo a tratar a un nińo de procedencia internacional, por el motivo que sea, deberá haber desarrollado el hábito de tratar a las personas de igual manera, independientemente de sus afinidades naturales, para no incurrir en una flagrante injusticia.

Finalmente, para garantizar una asistencia sanitaria competente y respetuosa con nińos de otras culturas, es decisiva la complicidad entre el médico y la enfermera. Cuando se actúa así se cuida la salud de estos niños y se avanza tanto en su integración social como en la de sus familias.

\section{Los deberes de la administración pública}

Hasta ahora me he referido a tres principios que deben informar la actuación de los profesionales sanitarios con los niños de procedencia internacional. Ahora bien, la garantía de que estos niños reciben la asistencia que necesitan no puede cargarse exclusivamente sobre ellos. La administración sanitaria tiene el deber de proporcionar los medios necesarios. Cuando ésta se encuentra con un nuevo desafío, como es la asistencia pediátrica a niños de procedencia internacional, será necesario que revise los medios disponibles y provea los convenientes en el caso de que los actuales resulten insuficientes o inadecuados.

Tanto la Declaración de Derechos de Niño de 1959 como la Convención de 1989 se refieren al derecho del niño a la salud. La primera en unos términos escuetos pero claros: "Tendrá derecho a crecer y desarrollarse en buena salud; con este fin deberán proporcionarse, tanto a él como a su madre, cuidados especiales, incluso atención prenatal y postnatal. El niño tendrá derecho a disfrutar de alimentación, vivienda, recreo y servicios médicos adecuados" (Principio 4). La segunda, de forma prolija y apelando a un concepto del derecho a la salud que, por más que haya sido consagrado por la OMS, es insostenible porque nadie puede garantizar el disfrute del más alto nivel posible de salud, como dice textualmente el art. 24 de la Convención, siguiendo lo dispuesto en el Preámbulo de la Constitución de la Organización Mundial de la Salud de 1948: "El goce del grado máximo de salud que se pueda lograr es uno de 
los derechos fundamentales de todo ser humano sin distinción de raza, religión, ideología política o condición económica o social". Pero más allá de este desafortunado inicio, el art. 24 contiene valiosas referencias para precisar el alcance del derecho a la salud de los niños de procedencia internacional. Concretamente, en el apartado 2.b se dice que habrá que "asegurar la prestación de la asistencia médica y la atención sanitaria que sean necesarias a todos los niños, haciendo hincapié en el desarrollo de la atención primaria de salud". Se trata pues de asegurar la asistencia a la totalidad de los niños y de prestar, además, una atención especial a la atención primaria.

Antes de hablar de medios concretos convendrá preguntarse por la cuestión acerca de la justa distribución de los recursos sanitarios. A la pregunta "¿qué nivel de asistencia sanitaria se debe ofrecer a los niños de procedencia internacional?” existen muy diversas respuestas. Aquí nos centraremos en las tres que podrían tener cabida dentro del marco constitucional español, dejando de lado otras alternativas que se dan en otros países pero que, en estos momentos, son impracticables en el nuestro:

- Se les debe permitir el acceso a los mismos recursos disponibles para el resto de la población, pero no se deben destinar recursos específicos para atender sus necesidades específicas.

- Tienen acceso a lo mismo que cualquier otro usuario y se procurará una cierta adaptación a sus necesidades, siempre que ello no suponga consumir recursos suplementarios.

- Se debe adaptar el sistema sanitario del país receptor a las necesidades de atención sanitaria de los inmigrantes, fundamentalmente a dos: las que tienen que ver con problemas de salud propios de esos colectivos y las derivadas de conseguir una adecuada comunicación con los pacientes. La primera garantiza una asistencia efectiva y la segunda el acceso a la asistencia. Ello supone destinar recursos, bien detrayéndolos de otras partidas de la asistencia sanitaria, o bien aportándolos ex novo al ámbito sanitario.

De estas tres respuestas, considero que lo correcto es optar por la tercera si es posible y, cuando no lo sea, al menos por la segunda. La cuestión es sumamente compleja: :por qué aceptar que se atiendan necesidades específicas de un colectivo y no se presten, en cambio, servicios que podrían beneficiar al conjunto de la población? ¿Por qué tiene más justificación financiar una red de mediadores culturales para atender a la población inmigrante que los empastes dentales de todos los usuarios de los servicios de salud? La justificación en este caso es clara: la mediación hace posible el acceso efectivo al sistema de salud, que es la condición fundamental para recibir asistencia sanitaria(15); los empastes mejoran notablemente la salud bucal, pero no son imprescindibles para desarrollar una vida con normalidad. No siempre el discernimiento de cada caso será sencillo: lo importante es que exista acuerdo sobre el principio, que consiste en garantizar a todos una asistencia sanitaria efectiva.

En todo caso, es vital que las políticas sanitarias que tienen que ver con los inmigrantes sean justas y que, en consecuencia, se pueda dar razón pública de ellas. De esta manera se garantizará a todos la misma asistencia sanitaria y se fomentará la integración de los inmigrantes como verdaderos ciudadanos.

Sin afán exhaustivo, señalaré algunas políticas y acciones de la administración sanitaria convenientes y, en algunos casos, imprescindibles:

- Facilitar el acceso efectivo a la atención sanitaria. Son muchas las intervenciones posibles en este campo. El espectro abarca desde medidas de sencilla aplicación y grandes resultados hasta otras costosas y superfluas. Lo razonable será incorporar las del primer tipo.

- Crear la figura de los mediadores culturales. La adecuada relación del profesional sanitario con los niños de culturas especialmente extrańas a la nuestra puede requerir de algo más que un intérprete cuando las partes no hablan una lengua común.

- Mayor seguimiento de los niños. Los niños de procedencia internacional pueden exigir un mayor seguimiento cuando, por razones culturales o económicas, no son llevados a los 
centros de salud periódicamente para recibir las vacunas o hacer las revisiones necesarias. Evidentemente, este último problema se da también con niños no inmigrantes, pero parece razonable que se plantee el modo de resolverlo para todos ellos.

- Formación de los profesionales que trabajan en las áreas más afectadas, tanto en los aspectos estrictamente sanitarios como socioculturales y, por supuesto, éticos(16).

- Recursos materiales necesarios. Las patologías de los niños de procedencia internacional serán, en la mayoría de los casos, comunes a las que puede sufrir un niño nacional. Pero cuando sean exclusivas de aquellos niños, será imprescindible disponer de los recursos sanitarios necesarios.

\section{Conclusión}

Los niños de procedencia internacional plantean un desafío a la asistencia pediátrica primaria. Para dar una respuesta satisfactoria, se deberían tener en cuenta los siguientes aspectos:
- Tan erróneo es caer en planteamientos paternalistas como autonomistas en la relación asistencial con estos niños.

- Tan erróneo es caer en el etnocentrismo, que desprecia el valor de las otras culturas en la asistencia sanitaria, como en el multiculturalismo, que consagra el valor absoluto de cualquier cultura.

- El médico y la enfermera deben mantener una permanente colaboración para asegurar la idónea asistencia sanitaria a estos niños.

- Los niños de procedencia internacional son especialmente vulnerables, necesitan amor y comprensión, y su asistencia sanitaria se ha de adaptar a sus necesidades reales.

- La administración pública tiene el deber de proveer los medios que garanticen la asistencia sanitaria idónea para los niños de procedencia internacional en igualdad de condiciones que a los nacionales. 
Ética de la asistencia primaria a los niños de procedencia internacional - Vicente Bellver Capella

\section{Referencias}

1. Allué Martínez X. La inmigración y su repercusión sanitaria: minorías étnicas y religiosas, exclusión social y nuevas enfermedades. En: De los Reyes M, Sánchez Jacob M. (eds.) Bioética y pediatría. Madrid: Ergon; 2010: 314-316.

2. Jansà JM, De Olalla P. Salud e inmigración: nuevas realidades y nuevos retos. Gaceta Sanitaria 2004; 18(1): 207-213.

3. Díaz Sierra, G. Problemas éticos en la consulta de Atención Primaria con población inmigrante. En Fátima Bermejo, F. Sanjuanbenito L. Ética y atención primaria. Madrid: Agencia Laín Entralgo; 2008: 231.

4. Martiniano Gómez, JL. Manual de medicina transcultural. Madrid: Semergen; 2003.

5. Bonet de Luna C. Bioética en pediatría. En AEPAP (ed.) Curso de actualización en pediatría. Madrid: ExLibris; 2004: 143.

6. Horno Goicoechea P. Necesidades afectivas de los niños, niñas y adolescentes en la atención primaria. En: De los Reyes M, Sánchez Jacob M. (eds.) Bioética y pediatría. Madrid: Ergon; 2010: 328.

7. Díaz Sierra G. Problemas éticos en la consulta de Atención Primaria con población inmigrante. En: De los Reyes M, Sánchez Jacob M. (eds.) Bioética y pediatría. Madrid: Ergon; 2010: 232.

8. Brykczynska GM. (ed.) Ethics in Paediatric Nursing. Londres: Chapman \& Hall; 1990.

9. Gasull Vilella M. Serret Serret M. Aspectos éticos de la enfermería pediátrica desde la perspectiva de la ética del cuidar. En: De los Reyes M, Sánchez Jacob M. (eds.) Bioética y pediatría. Madrid: Ergon; 2010: 647-653.

10. Rothman DJ. Strangers at the bedside: a history of how law and bioethics transformed medical decisionmaking. Nueva York: Basic Books; 1991.

11. Gracia D. Bioética y pediatría. En: De los Reyes M, Sánchez Jacob M. (eds.) Bioética y pediatría. Madrid: Ergon; 2010: 38.

12. Peiró AM, Vélez E, Calderón E, Martínez J. Reto bioético y legal de la asistencia sanitaria multicultural. Medicina Clínica 2007; 128(11): 438.

13. MacIntyre A. Animales racionales y dependientes. Por qué los seres humanos necesitamos las virtudes. Barcelona: Paidós; 2001.

14. Conill J, Cortina A. La fragilidad y la vulnerabilidad como partes constitutivas del ser humano. En: De los Reyes M, Sánchez Jacob M. (eds.) Bioética y pediatría. Madrid: Ergon; 2010: 39-48.

15. Burckhardt AQ, Revollo H, Collazos F, et al. La mediación intercultural sociosanitaria: implicaciones y retos. Norte de Salud Mental 2009: 35(4): 56-66.

16. Misra-Hebert A. Physician cultural competence: Cross-cultural communication improves care. Cleveland Clinic Journal of Medicine 2003: 70(4): 289-294.

Recibido: 4 de octubre de 2011

Aceptado: 18 de diciembre de 2011 